\title{
Alkaloids from Psychotria Target Sirtuins: In Silico and In Vitro Interaction Studies
}

Authors

Affiliations
Lionel Sacconnay ${ }^{1 *}$, Lucie Ryckewaert ${ }^{1 *}$, Carolina dos Santos Passos ${ }^{1}$, Maria Cristina Guerra ${ }^{2}$, Lucilia Kato ${ }^{3}$, Cecilia Maria Alves de Oliveira ${ }^{3}$, Amélia Henriques ${ }^{4}$, Pierre-Alain Carrupt ${ }^{1}$, Claudia Simões-Pires ${ }^{1}$, Alessandra Nurisso ${ }^{1}$

The affiliations are listed at the end of the article
Key words

alkaloids

- Psychotria spp.

- Rubiaceae

- sirtuins

- inhibitors

- docking

- molecular dynamics

received July 8,2014

revised Sept. 23, 2014

accepted October 6, 2014

Bibliography

Dol http://dx.doi.org/

10.1055/s-0034-1383261

Published online December 3,

2014

Planta Med 2015; 81: 517-524

(c) Georg Thieme Verlag KG

Stuttgart · New York .

ISSN 0032-0943

Correspondence

Dr. A. Nurisso

School of Pharmaceutical

Sciences

University of Geneva, University

of Lausanne

Quai Ernest-Ansermet 30

$\mathrm{CH}-1211$ Geneva 4

Switzerland

Phone: +41223793370

Fax: + 41223793360

Alessandra.Nurisso@unige.ch

Correspondence

Dr. Claudia Simões-Pires

School of Pharmaceutical

Sciences

University of Geneva, University

of Lausanne

Quai Ernest-Ansermet 30

CH-1211 Geneva 4

Switzerland

Phone: + 41223793370

Fax: + 41223793360

Claudia.Avello@unige.ch

\section{Abstract}

$\nabla$

Epigenetic enzymes such as histone deacetylases play a crucial role in the development of ageingrelated diseases. Among the 18 histone deacetylase isoforms found in humans, class III histone deacetylases, also known as sirtuins, seem to be promising targets for treating neurodegenerative conditions. Recently, Psychotria alkaloids, mainly monoterpene indoles, have been reported for their inhibitory properties against central nervous system cholinesterase and monoamine oxidase proteins. Given the multifunctional profile of these alkaloids in the central nervous system, and the fact that the indole scaffold has been previously associated with sirtuin inhibition, we hypothesized that these indole derivatives could al-

\section{Introduction}

\section{$\nabla$}

Sirtuins (SIRTs) are NAD+-dependent enzymes belonging to the class III histone deacetylase (HDAC) family. A total of seven SIRT human isoforms have been identified so far and classified into SIRT subclasses: Ia (SIRT1), Ib (SIRT2-3), II (SIRT4), III (SIRT5), and IV (SIRT6-7). In general, SIRTs are known to exert a deacetylase activity on the Nacetyl-lysine tails of histones and key cell signaling proteins, and have therefore been associated with the so-called epigenetic regulation [1]. In the specific case of neurodegenerative diseases, SIRTs have emerged as a potential pharmaceutical target as disease modifiers, since some of them have been found in functions related to ageing, memory, and neurodegenerative cascades. For instance, the activation of SIRT1, a predominantly nuclear enzyme, resulted in the proteolysis of amyloid precursor protein (APP) in the brain by

\footnotetext{
* These authors contributed equally to the work.
}

so interact with sirtuins. In the present study, alkaloids previously isolated from Psychotria spp. were evaluated for their potential interaction with human sirtuin 1 and sirtuin 2 by molecular docking and molecular dynamics simulation approaches. The in silico results allowed for the selection of five potentially active compounds, namely, prunifoleine, 14-oxoprunifoleine, E-vallesiachotamine, $Z$-vallesiachotamine, and vallesiachotamine lactone. The sirtuin inhibition of these compounds was confirmed in vitro in a dose-response manner, with preliminary information on their pharmacokinetics properties.

Supporting information available online at http://www.thieme-connect.de/products

triggering the transcription of the $\alpha$-secretase ADAM10. APP proteolysis by $\alpha$-secretase has been associated with neuroprotection in Alzheimer's disease in in vivo models, resulting in lower levels of $A \beta$ peptides, the precursors of senile plaques observed in this neurodegenerative condition [2-4]. On the other hand, the nonspecific SIRT inhibitor nicotinamide was able to restore cognitive deficits in a mouse model of Alzheimer's disease expressing both $A \beta$ peptide and tau protein features [5]. Both nicotinamide and sirtinol, another nonspecific SIRT inhibitor, were able to reduce IGF-1/IRS-2/Ras/ERK1/2 signalling and protect neurons [6]. Moreover, SIRT2 inhibition has been considered a promising target for neurodegenerative diseases. Indeed, ageing is one of the main risk factors for neurodegenerative diseases, and SIRT2 is a tubuline deacetylase, predominantly found in the cytoplasm, which has been shown to accumulate in the ageing brain [7]. The role of SIRT modulation in various neurodegenerative diseases has been extensively reviewed by others $[8,9]$. As part of our continuing interest in com- 
<smiles>COC(=O)C1=CN2CCc3c([nH]c4ccccc34)C2CC1C1=CCOC1=O</smiles><smiles>C=CC1CO[C@H]2Cc3c([nH]c4ccccc34)C[C@@H]12</smiles>

4<smiles>C/C=C(/C=O)C1CC2c3[nH]c4ccccc4c3CCN2C=C1C(=O)O</smiles>

2

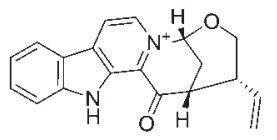

5<smiles>C/C=C(/C=O)[C@H]1C[C@@H]2c3[nH]c4ccccc4c3CCN2C=C1C(=O)OC</smiles>

3

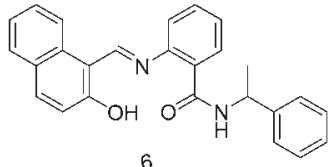

Fig. 1 Chemical structures of the Psychotria alkaloids vallesiachotamine lactone (1), E-vallesiachotamine (2), Z-vallesiachotamine (3), prunifoleine (4), and 14-oxoprunifoleine (5). The chemical structure of the nonspecific SIRT inhibitor sirtinol (6) is also shown. pounds with multifunctional biological activities targeting the central nervous system (CNS) $[10,11]$, indole alkaloids from Psychotria spp. (Rubiaceae) have previously been studied for their inhibitory activity on acetyl and butyrylcholinesterases (AChE and $\mathrm{BuChE}$, respectively), and monoamine oxidases $\mathrm{A}$ and $\mathrm{B}$ (MAO-A and MAO-B, respectively) [12-14]. The monoterpene indole alkaloids vallesiachotamine lactone (1; $\odot$ Fig. 1), E-vallesiachotamine (2; ○ Fig. 1), and Z-vallesiachotamine (3; ○ Fig. 1) isolated from Psychotria laciniata were BuChE and MAO-B inhibitors, while pauridianthoside (Fig. 1S, Supporting Information) inhibited MAO-A, and lyaloside (Fig. 1S, Supporting Information) was a BuChE inhibitor. The alkaloids prunifoleine (4; $\bullet$ Fig. 1$)$ and 14-oxoprunifoleine (5; $\odot$ Fig. 1) from Psychotria prunifolia were shown to inhibit $\mathrm{AChE}$ and MAO-A with $\mathrm{IC}_{50}$ values $\leq 10 \mu \mathrm{M}$, and were also able to inhibit BuChE and MAO-B at higher concentrations. Finally, strictosamide (from P. laciniata), vincosamide (from Psychotria leiocarpa), strictosidinic acid (from Psychotria myriantha), brachycerine (from Psychotria brachyceras), and psychollatine (from Psychotria umbellata) (Fig. 1S, Supporting Information) were not able to inhibit these enzymes [14]. Considering the multifunctional in vitro effects displayed by these indole alkaloids from Psychotria on targets related to neurodegeneration, and considering the potential SIRT inhibition by compounds possessing an indole scaffold [15], the capacity of the abovementioned alkaloids to bind SIRTs is herein investigated by following a computational structure-based approach. Enzymatic and cellbased results are also presented for corroborating the computational data in order to bring to light SIRT inhibition as a new mechanism of action of some of these multi-target natural products.

\section{Results}

$\nabla$

By taking into account crystallographic information of the productive forms suitable for docking studies [16], structural features of human SIRT1 and SIRT2 were analysed and compared. These proteins, sharing 39\% of sequence identity, are the closest isoforms among class III HDACs [17]. This identity increases up to $70 \%$ when the catalytic area, composed by the A-C subpockets, is considered. The two active sites, superimposed in 0 Fig. 2, presented similar volume cavity values $\left(1554 \AA^{3}\right.$ and $1638 \AA^{3}$, respectively) [18] and only punctual differences among residues. Whereas Arg466 in SIRT1 is replaced by Lys287 in SIRT2, preserving the physicochemical properties of this area in both isoforms, the electropositive Lys444 from SIRT1 is replaced by the polar but non-charged Gln265 in SIRT2 (๑ Fig. 2). The redocking of NAD ${ }^{+}$in

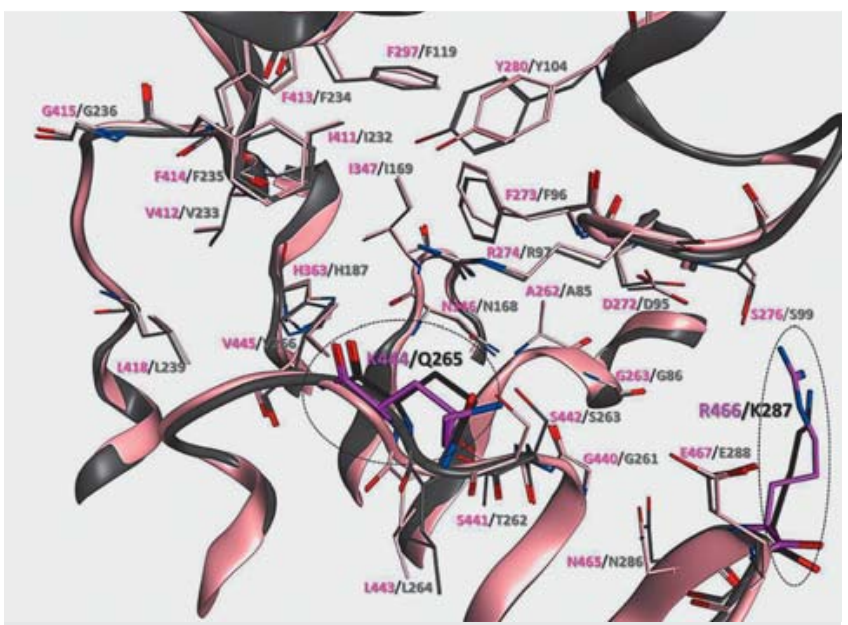

Fig. 2 Residues characterizing the two SIRT catalytic pockets are represented in capped sticks and colored in rose (SIRT1) and gray (SIRT2). Punctual differences between the two proteins are highlighted. (Color figure available online only.)

SIRT1 [19] demonstrated that the GOLD genetic algorithm was able to reproduce the crystallographic complex in silico, with RMSD values lower than $1.5 \AA$ (Fig. 2SA, Supporting Information). Interestingly, the same interaction network was retrieved for $\mathrm{NAD}^{+}$in SIRT2 (Fig. 2SB, Supporting Information). Differences in terms of PLP score, slightly lower in the SIRT2 pocket, were observed. Indeed, this may be due to the fact that the SIRT1 pocket, which co-crystallized with the co-substrate and is considered a rigid body during docking calculations, presented side chain conformations optimized for $\mathrm{NAD}^{+}$accommodation [19]. SIRT2, crystallized in the complex with ADP-ribose [20], characterized by a different organization of the $C$ subpocket, influenced the binding of the nicotinamide moiety predicted by docking at this specific site, leading to lower PLP values (Fig. 2SB, Supporting Information).

After the successful validation step, the docking of the 12 Psychotria alkaloids shown in Fig. 1S (Supporting Information) was then carried out and their binding mode evaluated by visual inspection, also taking into account the PLP score values as a qualitative criterion for understanding the potential strength of interaction. All these compounds shared a tetrahydro- $\beta$-carboline or a $\beta$-carboline scaffold: chemical modifications and substitutions on the scaffold are expected to determine whether the different binding behavior and affinities with the two targets is possible. It was 


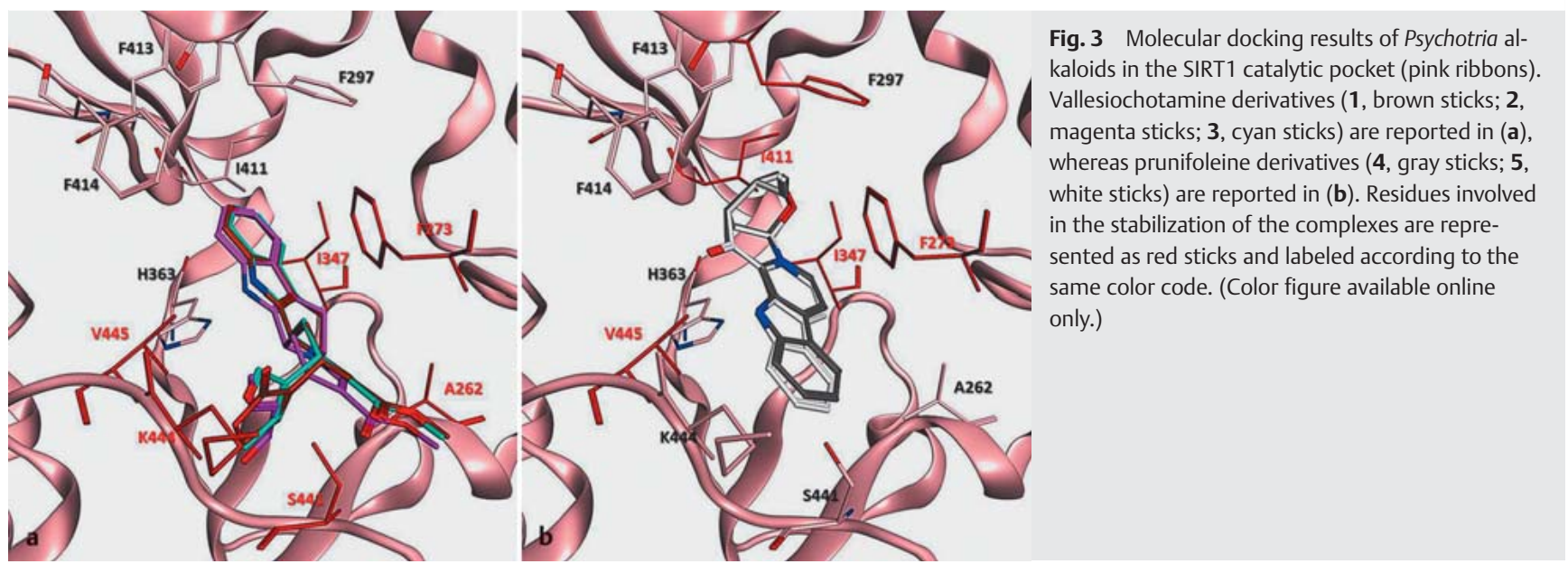

pointed out by the docking that the presence of a $\beta$-glucopyranosyl moiety determines an unfavorable binding mode, as also suggested by the negative/low PLP scores (Table 1S, Supporting Information). The steric hindrance created by this relatively rigid moiety prohibited the accommodation of compounds listed in Fig. 1S (Supporting Information) in the hydrophobic SIRT pockets. Despite these first negative results, this study showed that vallesiachotamine and prunifoleine derivatives presented a favorable steric complementarity with the proteins, with a conserved orientation of their tetrahydro- $\beta$-carboline and $\beta$-carboline scaffolds, respectively. Indeed, according to 0 Fig. 2 , it's not surprising that these ligands could bind both SIRT isoforms in a similar way. The common cyclic moiety accommodated in the SIRT1 hydrophobic niche was composed of Ala262, Phe273, Phe297, Ile347, and Val445 residues (@ Fig. 3). In the SIRT1-Zvallesiachotamine (3) complex, two additional hydrogen bonds were observed: the acetyl group of the natural chemical interacted with the Ser442 backbone, as also observed for E-vallesiachotamine (2). Moreover, the carbonyl group of compound 3 interacted with the Lys444 lateral chain. The same polar network was retrieved for vallesiachotamine lactone (1; 0 Fig. $\mathbf{3 a}$ ) whereas, for the prunifoleine derivatives ( 4 and $\mathbf{5}$ ), no polar contacts were detected ( Fig.3b). Docking results in the SIRT2 pocket (Fig.3S, Supporting Information) highlighted Ala85, Phe96, Ile69, Phe119, and Val266 as key residues for alkaloid binding. Polar interactions involving 3 were mediated by Gln265 and Ser263, with the latter also being involved in the stabilization of 1. No more contacts were found between alkaloids 2,4 , and 5 and SIRT2. According to these structural analyses (Fig. 4S and 5S, Supporting Information), the five selected alkaloids ( $\bullet$ Fig. 1 ) seem to behave as potential nonselective SIRT binders. Molecular docking for sirtinol (6; - Fig. 1), a pan-SIRT inhibitor, suggested that this compound fit into both SIRT pockets through nonpolar interactions, showing slightly higher PLP score values with respect to the docked alkaloids (Table 1S, Fig. 2S C and D, Supporting Information). With the PLP score being a qualitative parameter to evaluate the interaction strength of a complex, and docking results being a fast but an approximate way to predict interactions between biological partners, molecular systems obtained by docking were embedded in a box of explicit water molecules and their molecular and energetic features were monitored as a function of time through MD simulations. During the first $800 \mathrm{ps}$, energy, pressure, temperature, and density values reached a plateau, indicating a successful equilibration of all the molecular systems in water (Fig. 6S and 7S, Supporting Information). This stable behavior also characterized the production phase; no unexpected fluctuations were detected in the complexes, as indicated by the low RMSD and standard deviation (SD) values $(<1.5 \AA)$ of ligand atoms and residues characterizing the catalytic pockets (Table 2S, Supporting Information). In line with these observations, the key interactions previously highlighted by docking were kept along simulations (data not shown). MM-GBSA analyses were successively carried out for estimating the free energy of binding $\Delta \mathrm{G}_{\mathrm{MM}-\mathrm{GBSA}}$ of the complexes, using 1000 snapshots extracted from each production phase. The resulting negative energies indicated a favorable binding mode, with higher affinities for vallesiachotamine derivatives (1-3; - Fig. 4). Whereas energetic values associated to 3-5 were in the same range in the two enzymatic isoforms, compounds $\mathbf{1}$ and $\mathbf{2}$ showed slightly lower energies in SIRT1 with respect to SIRT2. This can be due to the missing polar network between SIRT2 and the alkaloids $\mathbf{1}$ and $\mathbf{2}$, stabilized only by hydrophobic interactions. The reference compound $\mathbf{6}$ showed a $\Delta \mathrm{G}_{\mathrm{MM}-\mathrm{GBSA}}$ value close to that obtained for vallesiachotamine derivatives, indicating a similar mechanism of action for these natural products related to a nonspecific SIRT inhibition.

In order to confirm the theoretical results, in vitro enzymatic assays were conducted on SIRT1. Enzymatic activity was determined by measuring the amount of deacetylated substrate generated in the enzymatic assay at different concentrations (10, 50, 100 , and $150 \mu \mathrm{M})$. The inhibitory profile of these alkaloids was comparable to 6 in a dose-response manner ( $\bullet$ Fig. 5). Alkaloids 1 and 5 were significantly $(\mathrm{p}<0.05)$ less potent than 6 at the highest concentrations ( 100 and $150 \mu \mathrm{M}$ ). This weaker inhibitory potential may be linked to solubility issues.

We have previously demonstrated that the nonspecific SIRT inhibition by 6 induces higher cytotoxicity than the specific SIRT1 inhibition in HEK 293 and HeLa cells [21]. In this study, compounds 2 and 3 displayed a reduced viability of HEK 293 cells comparable to 6 at the tested concentrations (Fig. 8S, Supporting Information), which was in line with the nonselective SIRT mechanism of inhibition hypothesized for $\mathbf{1 - 5}$. For compounds 1 and $\mathbf{4}$, it was not possible to observe a dose response, since they did not show enough cytotoxicity. By analyzing their structural features ( Fig. 1), it was possible to note that $\mathbf{4}$ and $\mathbf{5}$ are charged at a physiological $\mathrm{pH}$, whereas lactones were reported to be potentially unstable upon slight $\mathrm{pH}$ changes in biological cultures, enabling the opening of the lactone cycle and the formation of a de- 

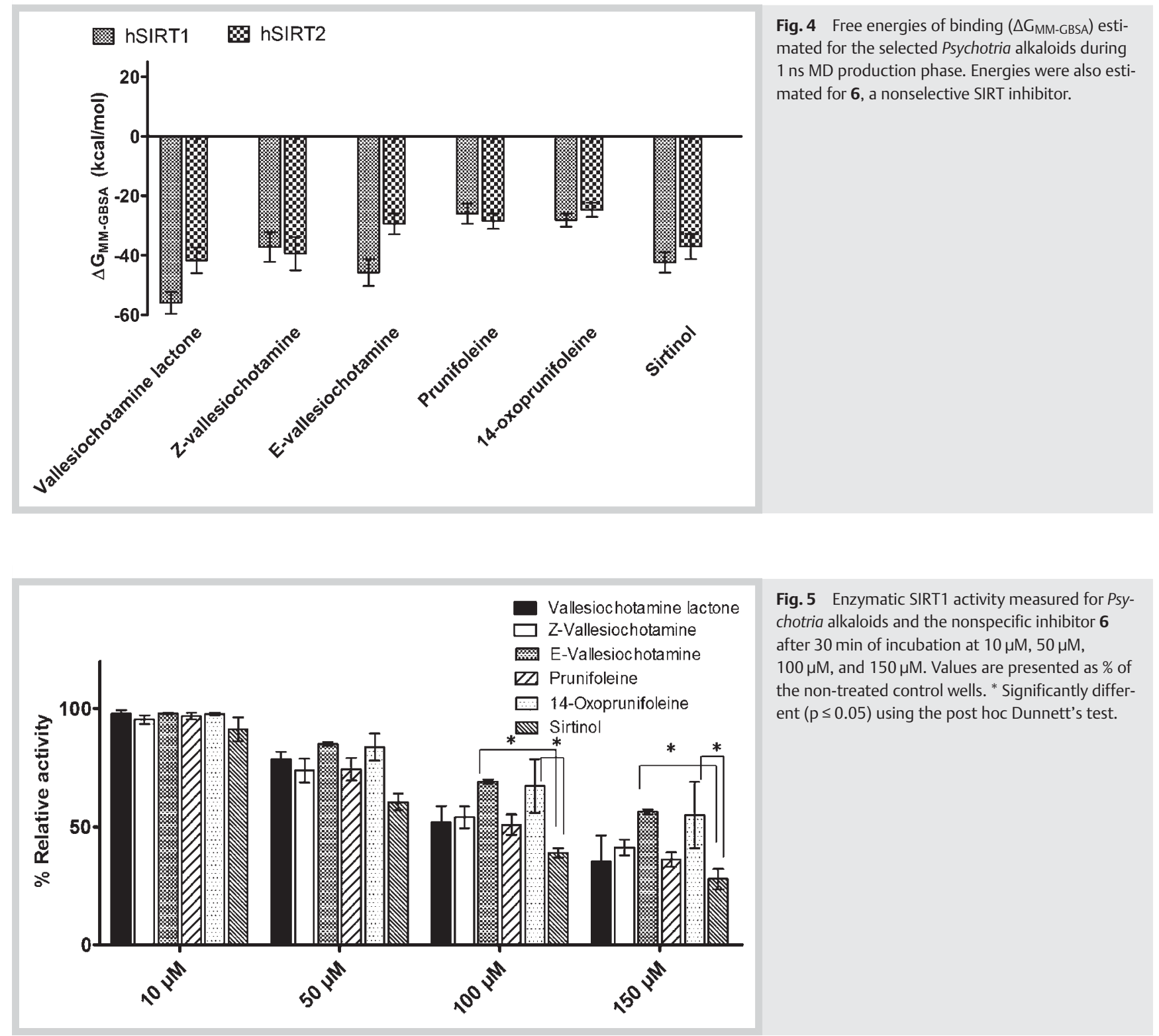

protonated carboxylic form [22]. It is known that charged compounds typically suffer from low cellular permeability. This may explain the missing cytotoxicity detection for these alkaloids. By applying an in-house passive blood brain barrier (BBB) permeability model developed in MOE (Chemical Computing Group, available upon request), it was possible to highlight the capability of all the compounds, with the exception of $\mathbf{1 , 4}$, and $\mathbf{5}$, to cross the barrier. Indeed, for having a central effect, an ideal neurotherapeutic agent should cross the BBB. In order to check the cytotoxic effects of these alkaloids in cells from the CNS, the effects on the viability and integrity of astrocyte cells submitted to treatments with $\mathbf{2}$ and $\mathbf{4}$ were evaluated by the MTT reduction, neutral red (NR) uptake, and propidium iodide (PI) exclusion assays. The tested concentrations were chosen based on the results verified in the SIRT enzymatic assays. As expected, viability and integrity experiments revealed that $\mathbf{4}$ did not impair the viability and integrity of astrocyte cells. This may be due to the low cell permeability of the compound as discussed previously. Nevertheless, $\mathbf{2}$ impaired the cell viability and integrity in the MTT ( Fig. 6 a), NR
( Fig. 6b), and PI (Fig. 9S, Supporting Information) assays when evaluated at $100 \mu \mathrm{M}$.

\section{Discussion}

$\nabla$

Neurodegenerative disorders such as Parkinson's disease (PD) and Alzheimer's disease (AD) are the result of multiple physiopathological processes regulating the neurodegenerative cascade. For this reason, multi-target drug candidates are of great interest for the treatment of such diseases [11,23]. It is known that nature is a rich source of phytochemicals possessing multifunctional properties [24]. Considering the inhibitory effects described for alkaloids from Psychotria on cholinesterases and monoamine oxidases [12-14] and the role of these enzymatic targets on the CNS and in neurodegenerative conditions [25], the present work proposed the investigation of the inhibitory activity of 12 Psychotria alkaloids ( Fig. 1; Fig. 1S, Supporting Information) against SIRT isoforms 1 and 2, recently identified as potential targets to fight against neurodegeneration [9]. First, a molecular docking study 


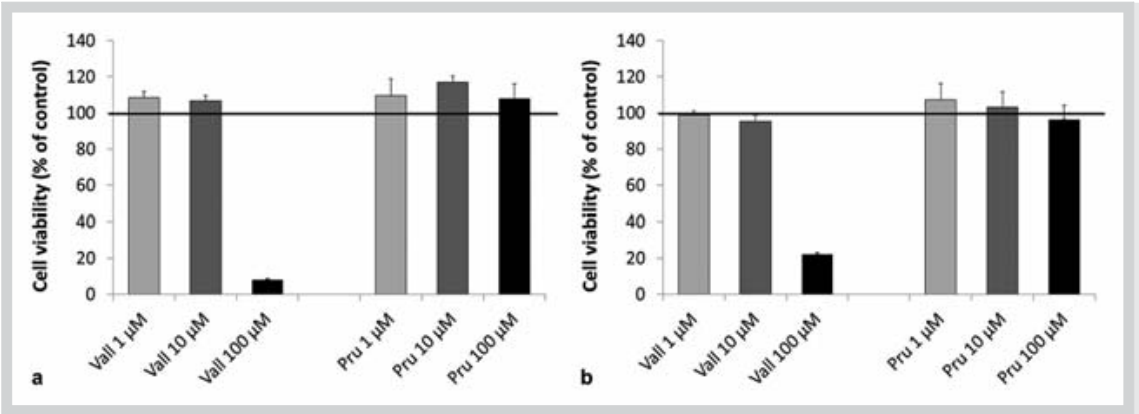

Fig. 6 Cytotoxic effects of alkaloids $\mathbf{2}$ and $\mathbf{4}$ on primary astrocytes cell cultures. Both alkaloids were tested in concentrations of 1,10 , and $100 \mu \mathrm{M}$ and incubated for $24 \mathrm{~h}$. Cellular viability was accessed by MTT reduction (a) and neutral red uptake (b) assays. Each value is the mean \pm SEM of three independent experiments performed in triplicate. ${ }^{*}$ Significantly different $(p \leq 0.05)$ using the Student's t-test.

was carried out to verify possible interactions of these phytochemicals with the two enzymatic targets. Computational results suggested a favorable binding mode for the non-glycoside monoterpene indole alkaloids ( $\bullet$ Fig. 1). Such compounds, as the nonselective SIRT inhibitor $\mathbf{6}$, occupied the catalytic B-C subpockets of the two isoforms in a similar way, and were mainly stabilized through hydrophobic contacts. This structural information suggested a competitive mechanism of inhibition for the five alkaloids; their position in the pockets would prevent NAD ${ }^{+}$binding. This was not the case for the synthetic indole inhibitor EX527, for which crystallographic details are available [19]. The latter, occupying part of the $C$ subpocket of SIRT1, allowed the simultaneous presence of $\mathrm{NAD}^{+}$and revealed a novel mechanism of inhibition (Fig. 10S, Supporting Information) [19]. 1 ns MD simulations allowed for the relaxation of the complexes in a hydrated environment. The molecular systems demonstrated certain stability over time, with low RMSD fluctuations (Table 2S, Supporting Information). Thermodynamic calculations confirmed the favorable binding of the five alkaloids, suggesting a higher affinity for 1-3 at the SIRT1 binding site, probably due to the presence of the additional polar contacts retrieved by docking and maintained during the simulations ( Fig. 4). In order to confirm these hypotheses, enzymatic and cellular assays were carried out. The five compounds selected in silico demonstrated a SIRT1 inhibitory profile comparable to that of $\mathbf{6}$ ( Fig. 5). As cytotoxicity is one of the mechanisms associated with nonselective SIRT inhibition, $\mathrm{IC}_{50}$ values on HEK 293 and on rat astrocyte primary cells were estimated. Only alkaloids $\mathbf{2}$ and $\mathbf{3}$ were able to impair the cell viability and integrity of both cellular lines at low (HEK 293) and high (astrocytes) concentrations ( Fig. 6; Fig. 85 and 9S, Supporting Information). These findings were in line with a recent study in which 2 was evaluated for its effects on cell viability and cell death on cultured human melanoma cells SK-MEL-37. Treatment of melanoma cells with this compound $(50 \mu \mathrm{M})$ for $24 \mathrm{~h}$ caused extensive cytotoxicity and necrosis [26]. The quaternary $\beta$-carbolines $(\mathbf{4}, \mathbf{5})$, as well as compound $\mathbf{1}$, demonstrated no apparent toxicity. This behavior may be due to their physicochemical properties leading to poor cell permeability, as also confirmed by a passive BBB prediction test conducted in silico.

The present work demonstrated for the first time the inhibitory properties of monoterpene indole alkaloids from Psychotria on SIRT enzymes, identified first through an in silico driven approach and then confirmed experimentally. Such evidences further prove the multifunctional effects of Psychotria alkaloids on the CNS, whose scaffold could be considered not only for the development of novel compounds with central activities, but also for the conception of chemical probes for SIRT target validation in neurodegeneration.

\section{Materials and Methods \\ $\nabla$}

Protein and ligand structures preparation

Crystallographic structures of the productive forms of human SIRT1 (PDB ID: 4I5I) and SIRT2 (PDB ID: 3ZGV) were retrieved from the Protein Data Bank (wwPDB; http://www.wwpdb.org/) $[19,20]$. The missing loop in SIRT2 was modelled according to our previously published protocol [21]. Both isoform structures were then prepared for structural inspection and docking in MOE 2012.10 by removing the crystallized water molecules and ligands, and by adding the missing hydrogen atoms. Three-dimensional structures of sirtinol (6) and the alkaloids of interest ( Fig. 1; Fig. 1S, Supporting Information) were constructed in MOE and their initial conformation optimized using the Amber force field $[27,28]$. Stereochemistry for all ligands was carefully checked, and the protonation state at $\mathrm{pH} 7.4$ was assigned using the Protonate 3D module of MOE [29].

\section{Molecular docking}

The molecular docking protocol was developed by using GOLD, version 5.2 (CCDC). SIRT1 and SIRT2 pockets were defined by considering those residues $13 \AA$ far from the $\mathrm{C} \beta$ atom of Ala262Ala85, respectively. The docking methodology was first checked and validated through the redocking of the inhibitor Ex-527 and the co-substrate $\mathrm{NAD}^{+}$into the SIRT1 pocket (PDB ID: 4I5I). The latter compound was then docked in SIRT2. Interactions between the two isoforms and compounds listed in 0 Fig. 1 and Fig. 1S (Supporting Information) were successively evaluated by applying the same protocol. One hundred docking poses for each flexible ligand, obtained by using the Preset options for the GOLD genetic search algorithm, were evaluated and ranked according to the PLP score. Based on both score and visual inspection analyses, alkaloids were selected for further in silico investigations.

\section{Molecular dynamics simulations}

Docking poses for the selected ligands were used as a starting point for explicit water molecular dynamic (MD) simulations. All complexes were prepared using the xleap module of Amber 12 [27]. Parameters for protein and ligands were taken from Amberff99SB [30] and Gaff [31] force fields, respectively. The AM1BCC method was used to calculate partial charges for ligands through the antechamber module of Amber 12. Each complex was then embedded in an octahedral box of TIP3P water molecules (10 ̊ around the solute) [32], neutralized by $\mathrm{Na}^{+}$ions, and minimized along 1000 steps with restraints on the solute using the pmemd module of Amber 12. Complexes were then free to relax along a further 1500 steps of conjugate gradient and 1000 steps of steepest descent energy minimization cycles. $100 \mathrm{ps}$ of slow heating at a constant volume (from $10 \mathrm{~K}$ to $300 \mathrm{~K}$ ) with 
weak restraints on the solute $(10.0 \mathrm{kcal} / \mathrm{mol} / \AA)$ was applied to all systems, followed by 100 ps MD at constant pressure conditions (1 atm). Equilibrium was reached for all systems after 600 additional ps monitoring, with the ptraj module of Amber12, energies, pressure, temperature, density, and RMSD values [27]. Trajectories were then collected each 2 ps along 1 ns of the MD production phase. The Particle-Mesh-Ewald algorithm (PME) with a cutoff of 8 Å was used for treating the long-range electrostatic effects [33] along with SHAKE algorithm to constrain the bonds connecting hydrogen atoms [34].

\section{Molecular dynamics trajectories analysis}

MD trajectories were analyzed with the ptraj module of Amber 12 through the calculation of hydrogen bonds and hydrophobic contact occupancies together with root mean square deviation (RMSD) values, by considering the initial equilibrate structure extracted from each MD production phase as a reference. Binding free energies $(\Delta G)$ between the ligands and each isoform were calculated by using an MM-GBSA [27] single trajectory approach. According to this method, solute internal energy contribution is estimated using the molecular mechanics force field, whereas the electrostatic and the nonpolar solvation energy terms are calculated by applying the generalized Born [35] and the solvent accessible surface area (SASA)-based models [36], respectively. Here, 1000 snapshots taken every 1 ps were extracted along the production phase and free energies of binding were estimated.

\section{Chemicals}

The five Psychotria indole alkaloids selected from the in silico workflow were obtained from isolation procedures described previously, after which they have been kept at $-20^{\circ} \mathrm{C}$ as part of our in-house chemical library for natural products. Vallesiachotamine lactone (1, purity $>90 \%$ ) and $E / Z$-vallesiachotamine compounds ( $\mathbf{2}$ and $\mathbf{3}$, purity $>95 \%$ for both compounds) were isolated from P. laciniata Vell., collected from the Atlantic forest of Cocal do Sul (Santa Catarina, Brazil S26 80'; W48 ${ }^{\circ} 5^{\prime}$; ICN 182552 , Herbarium of the Federal University of Rio Grande do Sul). Briefly, the dried leaves were extracted with EtOH at room temperature. The solvent was removed under vacuum and the residual extract dissolved in $1 \mathrm{~N} \mathrm{HCl}$ and exhaustively extracted with $\mathrm{CH}_{2} \mathrm{Cl}_{2}$, in order to remove non-polar constituents. Subsequently, the acid extracts were alkalinized with $25 \% \mathrm{NH}_{4} \mathrm{OH}$ (pH 9-10) and partitioned with $\mathrm{CH}_{2} \mathrm{Cl}_{2}$, resulting in the P. laciniata alkaloid-enriched extract. This extract was fractionated by reversed phase medium pressure liquid chromatography (RP-MPLC) with a $\mathrm{H}_{2} \mathrm{O}-\mathrm{CH}_{3} \mathrm{CN}$ gradient step, Fractions containing $\mathbf{1 ,} \mathbf{2}$ and $\mathbf{3}$ were submitted to preparative HPLC with isocratic elution $\left(\mathrm{H}_{2} \mathrm{O}\right.$ : $\mathrm{CH}_{3} \mathrm{CN}, 40: 60, \mathrm{v} / \mathrm{v}$ ), rate of $5 \mathrm{~mL} / \mathrm{min}$, and detection at $280 \mathrm{~nm}$, affording the isolated compounds [14]. Prunifoleine and 14-oxoprunifoleine (4 and 5, purity $>90 \%$ for both compounds) were isolated from $P$. prunifolia (Kunth) Steyerm., collected from the seasonal semi-deciduous forest in Goiânia (Goiás, Brazil, S16 ${ }^{\circ} 36^{\prime}$ 12 , W49 ${ }^{\circ} 15^{\prime}$, Delprete 10323 , Herbarium of the Federal University of Goiás). Briefly, an alkaloid-enriched extract was obtained as described for P. laciniata, and compounds were isolated by repeated column chromatography on silica gel $60\left(\mathrm{CHCl}_{3}-\mathrm{MeOH}-\right.$ $\mathrm{NH}_{4} \mathrm{OH}$ eluent system in gradient form) and successive purification by preparative TLC on silica gel with $\mathrm{CHCl}_{3} / \mathrm{MeOH}(25: 75)$ [37]. Sirtinol (purity $>98 \%$, Santa Cruz Biotechnology), ammonium chloride (purity $>99.5 \%$, Sigma-Aldrich) and hydrogen peroxide ( $50 \%$ stabilized solution, Sigma-Aldrich) were used as positive controls. Prior to enzymatic and cell-based experiments, all alkaloids had their purity assessed by UHPLC/HR-TOF-MS and NMR spectroscopy.

\section{UHPLC/HR-TOF-MS analyses}

UHPLC/HR-TOF-MS analyses were performed on a Micromass-LCT Premier time-of-flight mass spectrometer from Waters with an electrospray (ESI) interface coupled with an Acquity UPLC system from Waters. Detection was performed in the positive ion mode in the range of $m / z 100-1000$ in the centered mode with a scan time of $0.2 \mathrm{~s}$ and an interscan delay of $0.3 \mathrm{~s}$ for polarity switching. ESI conditions were: capillary voltage $2800 \mathrm{~V}$, cone voltage $40 \mathrm{~V}$, source temperature $120^{\circ} \mathrm{C}$, desolvation temperature $250^{\circ} \mathrm{C}$, cone gas flow $20^{\circ} \mathrm{L} / \mathrm{h}$, and desolvation gas flow $800 \mathrm{~L} / \mathrm{h}$. For the internal calibration, a solution of leucine/enkephalin from Sigma-Aldrich at $5 \mu \mathrm{g} / \mathrm{mL}$ was infused through the lockmass probe at a flow rate of $5 \mu \mathrm{L} / \mathrm{min}$ using a second Shimadzu LC-10ADvp LC pump.

\section{NMR analyses}

NMR spectra were recorded on a Varian Inova $500 \mathrm{MHz}$ spectrometer. The instrument was controlled using Varian NMR software installed on a Sun SPARCstation. The chemical shifts are given in $\delta(\mathrm{ppm})$ with residual solvent signal as the internal reference. The coupling constants are given in Hz. Spectral data of the isolated alkaloids are given as Supporting Information (Table 3S).

\section{Enzymatic assays}

Reactions were carried out in 96 -well $1 / 2$ volume plates. For the SIRT1 enzymatic assay, a preincubation was conducted with $550 \mu \mathrm{M} \mathrm{NAD}^{+}, 30 \mu \mathrm{M}$ substrate (Fluor de Lys ${ }^{\mathrm{TM}}$ SIRT1, Enzo), and test samples ( $3 \%$ DMSO final concentration) at $37^{\circ} \mathrm{C}$ for $15 \mathrm{~min}$ under agitation. The enzymatic reaction was started by the addition of 1 unit/well of human recombinant SIRT1 enzyme (Sigma Aldrich) in assay buffer (50 mM Tris/ $\mathrm{HCl} \mathrm{pH} \mathrm{8.0,} 137 \mathrm{mM} \mathrm{NaCl}$, $2.7 \mathrm{mM} \mathrm{KCl}, 1 \mathrm{mM} \mathrm{MgCl} 2,1 \mathrm{mg} / \mathrm{ml} \mathrm{BSA}$ ), except for blank wells that received buffer and vehicle only. The plate was further incubated at $37^{\circ} \mathrm{C}$ for $30 \mathrm{~min}$ under agitation. The reaction was stopped by the addition of $1 \mathrm{mM}$ nicotinamide. Fluor de Lys developer II ${ }^{\mathrm{TM}}$ (BML-KI176) was diluted 5-fold in assay buffer and added to every well. The relative amounts of deacetylated substrate were obtained by fluorescence reading (FLx800 ${ }^{\mathrm{TM}}$, BioTek, excitation $360 \mathrm{~nm}$, emission $460 \mathrm{~nm}$ ). SIRT activity was calculated by comparing the amount of deacetylated substrate between the DMSO control (100\% SIRT activity) and the test sample. Sirtinol was used as a positive control.

\section{Cell culture}

HEK 293 cells (ATCC ${ }^{\circledR}$ CRL-1573 ${ }^{\mathrm{TM}}$ ) were cultured in minimum essential medium (MEM) $\alpha$, no nucleosides (Gibco), supplemented with $10 \%$ heat-inactivated FBS, 100 units/mL penicillin, and $100 \mu \mathrm{g} / \mathrm{mL}$ streptomycin. Cultures were kept in $75 \mathrm{~cm}^{2}$ tissue culture flasks (NUNC, Thermo Scientific) at $37^{\circ} \mathrm{C}$ in a $5 \% \mathrm{CO}_{2}$ incubator (HERAcell, Heraeus). Primary cortical astrocyte cultures from Wistar rats were prepared as previously described [38]. All procedures were in accordance with the NIH guidelines for the care and use of laboratory animals and were approved by the ethical committee from the host institution (CEUA, UFRGS, Porto Alegre, Brazil). Briefly, cortices of newborn Wistar rats (1-2 days old) were removed and mechanically dissociated in $\mathrm{Ca}^{2+}$ and $\mathrm{Mg}^{2+}-$ free balanced salt solution. The cortex was cleaned of meninges and mechanically dissociated by sequential passage through a 
Pasteur pipette. After centrifugation at $1000 \mathrm{rpm}$ for $5 \mathrm{~min}$, the pellet was resuspended in DMEM (pH 7.6) supplemented with 8.39 mM HEPES, 23.8 mM NaHCO 3 , 0.1\% Fungizone ${ }^{\circledR}, 0.032 \%$ garamicine, and $10 \%$ FCS. Cultures were maintained in DMEM containing $10 \% \mathrm{FCS}$ in $5 \% \mathrm{CO}_{2} / 95 \%$ air at $37^{\circ} \mathrm{C}$ and allowed to grow to confluence.

\section{Crystal violet cell viability assay}

Assays were performed in sterile 96-well flat bottom polystyrene TC-treated microplates. Microplate wells were previously coated with $50 \mu \mathrm{l} /$ well of $20 \mu \mathrm{g} / \mathrm{ml}$ poly-D-lysine in DPBS for $30 \mathrm{~min}$, washed with double distilled water, and let dry under laminar flow. HEK 293 cells were plated at 6400 cells/well for the 24 -h assay. Cells were allowed to attach for $6 \mathrm{~h}$ before treatment with compounds. Tested samples were diluted in DMSO leading to a $0.5 \%$ DMSO final concentration. After the incubation time $(24 \mathrm{~h})$, the medium was removed and $200 \mu \mathrm{L}$ of $0.2 \%$ crystal violet solution (in $2 \%$ ethyl alcohol) was added to each well, followed by 10 min incubation. After removal of the crystal violet solution, the wells were washed several times with tap water, and let to dry. Crystal violet dye was solubilized by the addition of $200 \mu \mathrm{L} /$ well of $0.5 \%$ SDS in 50\% ethyl alcohol. Microplates were read at $595 \mathrm{~nm}$ in a plate reader (ELX800 ${ }^{\mathrm{TM}}$, Bio-Tek). Cell cytotoxicity was calculated from a percent relative to the DMSO control. Sirtinol was used as a positive control [18].

\section{MTT reduction cell viability assay}

Viability was assayed via the colorimetric MTT method (Hansen et al., 1989). Briefly, the cells were incubated with $0.5 \mathrm{mg} / \mathrm{mL}$ MTT followed by incubation in $5 \% \mathrm{CO}_{2}$ at $37^{\circ} \mathrm{C}$ for $30 \mathrm{~min}$. The formazan product generated during the incubation was solubilized in DMSO. Absorbance values were measured at 560 and $630 \mathrm{~nm}$. The results are expressed as a percentage of the control. Ammonium chloride and hydrogen peroxide were used as positive controls.

\section{Neutral red uptake cell viability assay}

Astroglial cell viability was evaluated by the neutral red uptake assay. Briefly, rat cortical astrocytes were incubated for $24 \mathrm{~h}$ with the treatments: E-vallesiachotamine and prunifoleine at 1, 10, and $100 \mu \mathrm{M}$ prepared in DMEM medium. After this period, the neutral red reagent was added to the medium, and the cells were incubated for $30 \mathrm{~min}$. Then, the medium was completely removed and the wells were washed with PBS buffer $(2 \times 5 \mathrm{~min})$. Finally, after the homogenization of the well's content with an acid acetic solution, the colorimetric readings were performed at $560 \mathrm{~nm}$. The results are expressed as a percentage of the control. Ammonium chloride and hydrogen peroxide were used as positive controls.

\section{Propidium iodide assay}

Cellular damage was assessed by fluorescent image analysis of PI exclusion assay as previously described [39]. Cells were incubated with alkaloids E-vallesiachotamine (2) and prunifoleine (4) in concentrations corresponding to 1,10 , and $100 \mu \mathrm{M}$ for $24 \mathrm{~h}$. Concomitantly, PI $(7.5 \mu \mathrm{M})$ was added to the culture medium. After the incubation, cells were analyzed and photographed with a Nikon inverted microscope using a TE-FM epifluorescence accessory. Optical density was determined with the Optiquant version 02.00 software (Packard Instrument Company). All images are representative fields from three experi- ments carried out in triplicate. Ammonium chloride and hydrogen peroxide were used as positive controls.

\section{Statistical analysis}

Data are reported as the mean \pm SEM and were analyzed by oneway analysis of variance (ANOVA) followed by Duncan's test or by Student's t-test when indicated in the results section and performed in GraphPad Prism 5.0. Values of $\mathrm{p}<0.05$ were considered significant.

\section{Supporting information}

Docking and MD calculations, together with cell viability (HEK 293 and primary astrocyte cells) and spectral data, are available as Supporting Information.

\section{Acknowledgements}

We would like to thank Jean-Baptiste Haas for the conception and development of the passive BBB prediction tool used in this study, and the Professors Carlos Alberto Saraiva Gonçalves and Carmem Gottfried from the Biochemistry Department of the Federal University of Rio Grande do Sul for making the experiments with astrocyte cell cultures available. Alessandra Nurisso would like to gratefully acknowledge the Excellence Fellowship Programme of the University of Geneva, Switzerland, for financial support.

\section{Conflict of Interest}

$\nabla$

The authors declare no conflict of interest.

\section{Affiliations}

${ }^{1}$ School of Pharmaceutical Sciences, University of Geneva, University of Lausanne, Geneva, Switzerland

2 Departamento de Bioquímica, Instituto de Ciências Básicas da Saúde,

Universidade Federal do Rio Grande do Sul, Porto Alegre, RS, Brazil

${ }^{3}$ Laboratório de Bioatividade Molecular, Instituto de Química, Universidade Federal de Goiás, UFG, Goiânia, GO, Brazil

${ }^{4}$ Laboratório Farmacognosia, Departamento de Produção de Matéria-Prima, Faculdade de Farmácia, Universidade Federal do Rio Grande do Sul, Porto Alegre, RS, Brazil

\section{References}

1 Huber K, Superti-Furga G. After the grape rush: sirtuins as epigenetic drug targets in neurodegenerative disorders. Bioorg Med Chem 2011; 19: 3616-3624

2 Kojro E, Fahrenholz F. The non-amyloidogenic pathway: structure and function of alpha-secretases. Subcell Biochem 2005; 38: 105-127

3 Mattson MP. Cellular actions of beta-amyloid precursor protein and its soluble and fibrillogenic derivatives. Physiol Rev 1997; 77: 1081-1132

4 Postina R, Schroeder A, Dewachter I, Bohl J, Schmitt U, Kojro E, Prinzen C, Endres K, Hiemke C, Blessing M, Flamez P, Dequenne A, Godaux E, van Leuven F, Fahrenholz F. A disintegrin-metalloproteinase prevents amyloid plaque formation and hippocampal defects in an Alzheimer disease mouse model. J Clin Invest 2004; 113: 1456-1464

5 Green KN, Steffan JS, Martinez-Coria H, Sun X, Schreiber SS, Thompson $L M$, LaFerla FM. Nicotinamide restores cognition in Alzheimer's disease transgenic mice via a mechanism involving sirtuin inhibition and selective reduction of Thr231-phosphotau. J Neurosci 2008; 28: 1150011510

6 Li Y, Xu W, McBurney MW, Longo VD. SIRT1 inhibition reduces IGF-I/ IRS-2/Ras/ERK1/2 signaling and protects neurons. Cell Metab 2008; 8: 38-48

7 Maxwell MM, Tomkinson EM, Nobles J, Wizeman JW, Amore AM, Quinti L, Chopra V, Hersch SM, Kazantsev AG. The Sirtuin 2 microtubule deacety- 
lase is an abundant neuronal protein that accumulates in the aging CNS. Hum Mol Genet 2011; 20: 3986-3996

8 Dillin A, Kelly JW. The yin-yang of sirtuins. Science 2007; 317: 461-462

9 Donmez G, Outeiro TF. SIRT1 and SIRT2: emerging targets in neurodegeneration. EMBO Mol Med 2013; 5: 344-352

10 Nurisso A, Simoes-Pires C, Martel S, Cressend D, Guillot A, Carrupt PA. How to increase the safety and efficacy of compounds against neurodegeneration? A multifunctional approach. Chimia 2012; 66: 286-290

11 Novaroli L, Daina A, Bertolini F, Di Giovanni S, Bravo J, Reist M, Carrupt $P A$. Identification of novel multifunctional compounds for the treatment of some aging related neurodegenerative diseases. Chimia 2005; 59: $315-320$

12 Farias FM, Passos CS, Arbo MD, Barros DM, Gottfried C, Steffen VM, Henriques AT. Strictosidinic acid, isolated from Psychotria myriantha Mull. Arg. (Rubiaceae), decreases serotonin levels in rat hippocampus. Fitoterapia 2012; 83: 1138-1143

13 Dos Santos Passos C, Soldi TC, Torres Abib R, Anders Apel M, Simões-Pires $C$, Marcourt L, Gottfried C, Henriques AT. Monoamine oxidase inhibition by monoterpene indole alkaloids and fractions obtained from Psychotria suterella and Psychotria laciniata. J Enzyme Inhib Med Chem 2013; 28: 611-618

14 Passos CS, Simões-Pires CA, Nurisso A, Soldi TC, Kato L, de Oliveira CM, de Faria EO, Marcourt L, Gottfried C, Carrupt PA, Henriques AT. Indole alkaloids of Psychotria as multifunctional cholinesterases and monoamine oxidases inhibitors. Phytochemistry 2013; 86: 8-20

15 Napper AD, Hixon J, McDonagh T, Keavey K, Pons JF, Barker J, Yau WT, Amouzegh P, Flegg A, Hamelin E, Thomas RJ, Kates M, Jones S, Navia MA, Saunders JO, DiStefano PS, Curtis R. Discovery of indoles as potent and selective inhibitors of the deacetylase SIRT1. J Med Chem 2005; 48: 8045-8054

16 Sacconnay L, Angleviel M, Randazzo GM, Marcal Ferreira Queiroz M, Ferreira Queiroz E, Wolfender JL, Carrupt PA, Nurisso A. Computational studies on sirtuins from Trypanosoma cruzi: structures, conformations and interactions with phytochemicals. PLoS Negl Trop Dis 2014; 8: e2689

17 Costantini S, Sharma A, Raucci R, Costantini M, Autiero I, Colonna G. Genealogy of an ancient protein family: the Sirtuins, a family of disordered members. BMC Evol Biol 2013; 13: 60

18 Dundas J, Ouyang Z, Tseng J, Binkowski A, Turpaz Y, Liang J. CASTp: computed atlas of surface topography of proteins with structural and topographical mapping of functionally annotated residues. Nucleic Acids Res 2006; 34: W116-W118

19 Zhao X, Allison D, Condon B, Zhang F, Gheyi T, Zhang A, Ashok S, Russell M, MacEwan I, Qian Y, Jamison JA, Luz JG. The 2.5 A crystal structure of the SIRT1 catalytic domain bound to nicotinamide adenine dinucleotide $\left(\mathrm{NAD}^{+}\right)$and an indole (EX527 analogue) reveals a novel mechanism of histone deacetylase inhibition. J Med Chem 2013; 56: 963-969

20 Moniot S, Schutkowski M, Steegborn C. Crystal structure analysis of human Sirt2 and its ADP-ribose complex. J Struct Biol 2013; 182: 136143

21 Ryckewaert L, Sacconnay L, Carrupt PA, Nurisso A, Simões-Pires CA. Nonspecific SIRT inhibition as a mechanism for the cytotoxicity of ginkgolic acids and urushiols. Toxicol Lett 2014; 229: 374-380

22 Yates EA, Philipp B, Buckley C, Atkinson S, Chhabra SR, Sockett RE, Goldner M, Dessaux Y, Cámara M, Smith H, Williams P. N-acylhomoserine lactones undergo lactonolysis in a $\mathrm{pH}-$, temperature-, and acyl chain length-dependent manner during growth of Yersinia pseudotuberculosis and Pseudomonas aeruginosa. Infect Immun 2002; 70: 5635-5646
23 Cavalli A, Bolognesi ML, Minarini A, Rosini M, Tumiatti V, Recanatini M, Melchiorre C. Multi-target-directed ligands to combat neurodegenerative diseases. J Med Chem 2008; 51: 347-372

24 Efferth T, Koch E. Complex interactions between phytochemicals. The multi-target therapeutic concept of phytotherapy. Curr Drug Targets 2011; 12: 122-132

25 Geldenhuys WJ, Youdim MB, Carroll RT, Van der Schyf CJ. The emergence of designed multiple ligands for neurodegenerative disorders. Prog Neurobiol 2011; 94: 347-359

26 Soares PR, de Oliveira PL, de Oliveira CM, Kato L, Guillo LA. In vitro antiproliferative effects of the indole alkaloid vallesiachotamine on human melanoma cells. Arch Pharm Res 2012; 35: 565-571

27 Case DA, Darden TA, Cheatham III TE, Simmerling CL, Wang J, Duke RE, Luo R, Walker RC, Zhang W, Merz KM, Roberts B, Hayik S, Roitberg A, Seabra G, Swails J, Goetz AW, Kolossvary I, Wong KF, Paesani F, Vanicek J, Wolf RM, Liu J, Wu X, Brozell SR, Steinbrecher T, Gohlke H, Cai Q Ye X, Wang J, Hsieh MJ, Cui G, Roe DR, Mathews DH, Seetin MG, Salomon-Ferrer R, Sagui C, Babin V, Luchko T, Gusarov S, Kovalenko A, Kollman PA. Amber 12. San Francisco: University of California; 2012

28 Gerber PR, Muller K. MAB, a generally applicable molecular force field for structure modelling in medicinal chemistry. J Comput Aided Mol Des 1995; 9: 251-268

29 Labute P. Protonate3D: assignment of ionization states and hydrogen coordinates to macromolecular structures. Proteins 2009; 75: 187-205

30 Hornak V, Abel R, Okur A, Strockbine B, Roitberg A, Simmerling C. Comparison of multiple Amber force fields and development of improved protein backbone parameters. Proteins 2006; 65: 712-725

31 Wang J, Wolf RM, Caldwell JW, Kollman PA, Case DA. Development and testing of a general amber force field. J Comput Chem 2004; 25: $1157-$ 1174

32 Aaqvist J. Ion-water interaction potentials derived from free energy perturbation simulations. J Phys Chem 1990; 94: 8021-8024

33 Darden T, York D, Pedersen L. Particle mesh Ewald: An $N \cdot \log (N)$ method for Ewald sums in large systems. J Chem Phys 1993; 98: 10089-10092

34 Ryckaert JP, Ciccotti G, Berendsen HJC. Numerical integration of the cartesian equations of motion of a system with constraints: molecular dynamics of $n$-alkanes. J Comput Phys 1977; 23: 327-341

35 Onufriev A, Bashford D, Case DA. Exploring protein native states and large-scale conformational changes with a modified generalized born model. Proteins 2004; 35: 383-394

36 Case DA, Darden TA, Cheatham III TE, Simmerling CL, Wang J, Duke RE, Luo R, Walker RC, Zhang W, Merz KM, Roberts B, Wang B, Hayik S, Roitberg A, Seabra G, Kolossvary I, Wong KF, Paesani F, Vanicek J, Liu J, Wu X, Brozell SR, Steinbrecher T, Gohlke H, Cai Q Ye X, Wang J, Hsieh MJ, Cui G, Roe DR, Mathews DH, Seetin M G, Sagui C, Babin V, Luchko T, Gusarov S, Kovalenko A, Kollman PA. Amber 11. San Francisco: University of California; 2010

37 Faria EO, Kato L, de Oliveira CMA, Carvalho BG, Silva CC, Sales LS, Schuquel ITA, Silveira-Lacerda EP, Delprete PG. Quaternary $\beta$-carboline alkaloids from Psychotria prunifolia (Kunth) Steyerm. Phytochem Lett 2010; 3: 113-116

38 Gottfried C, Cechin SR, Gonzalez MA, Vaccaro TS, Rodnight R. The influence of the extracellular matrix on the morphology and intracellular $\mathrm{pH}$ of cultured astrocytes exposed to media lacking bicarbonate. Neuroscience 2003; 121: 553-562

39 Quincozes-Santos A, Nardin P, de Souza DF, Gelain DP, Moreira JC, Latini $A$, Gonçalves CA, Gottfried $C$. The janus face of resveratrol in astroglial cells. Neurotox Res 2009; 16: 30-41 